\title{
Doppler Shifted "In" and "Out" Longitudinal Matter Waves as the Carriers of Particle Relativistic Momentum and Energy
}

\author{
Dan Wagner ${ }^{1}$ \\ ${ }^{1} 15$ Bradley Road, Madison, CT 06443 \\ Correspondence: Dan Wagner, 15 Bradley Road, Madison, CT 06443, USA. E-mail: drwagner10@comcast.net
}

Received: March 10, 2020

Accepted: March 252020

Online Published: May 27, 2020

doi:10.5539/apr.v12n3p11

URL: http://dx.doi.org/10.5539/apr.v12n3p11

\begin{abstract}
Momentum and Kinetic Energy equations are developed from the hypothesis that oppositely directed components of harmonically oscillating pseudo standing waves pass through a quantum particle center and can be represented by Longitudinal Matter Waves that carry the particle's momentum and energy. The Doppler effect on the component wave lengths allows the net forward momentum and kinetic energy to increase with speed well beyond classical values. De Broglie (1925) issues with stationary wavelength and moving pulse rate are resolved in a different manner.

Because a quantum particle is considered to be nothing more than the sum of "in" and "out" matter waves focused through its center, whatever happens to these matter waves determines the future location of that center. This opens the door to physical explanations for gravity, interference, and the slowdown of light in transparent mediums. Gravity, for example, is shown in section 6 , to possibly be caused by the local gradient in matter wave speed near a large body like earth.

Keywords: Longitudinal Matter Wave, Sound Wave Model, Matter Wave Momentum, Doppler Effect, Total Internal Momentum, Net Forward Momentum, Kinetic Energy, Rest Energy, Preferred Reference Frame, Lorentz-Poincare' Physical Viewpoint, Gravity, Matter Wave Gradient
\end{abstract}

\section{Introduction}

Commenting on the theory of quanta in his original doctoral thesis, Louis De Broglie (1925 ) says "One may imagine that, by cause of a meta law of Nature, to each portion of energy with a proper mass, $m_{0}$, one may associate a periodic phenomenon of frequency $v_{0}, \ldots . .$. . He also says "Must we suppose that this periodic phenomenon occurs in the interior of energy packets? This is not at all necessary; the results of paragraph 1.3 will show that it is spread out over an extended space." This viewpoint led him to the very successful momentum relationship $p=h / \lambda$ for all basic particles, where $h$ is Plank's constant, $\lambda$ is the measured wavelength, and $p$ is momentum. Despite the tremendous success of this relationship, there is still a troubling aspect to it. When the momentum is zero (i.e. when the particle is "stationary"), the wavelength must be infinite, yet a stationary frequency is defined by De Broglie's energy relationship. What initially also troubled him was that based on Special Relativity, the predicted change in frequency of a vibration, as speed increases, is the opposite of what one might expect based on increased energy and his analogy between particles and photons, where energy $=h x$ frequency, and higher (not lower) frequency means higher energy.

Our goal is to develop a quantum particle model which is consistent with experimental results for momentum and energy vs speed but;

1) does not require an infinite stationary wavelength

2) can accommodate a speed based physical pulse rate which is different from the electromagnetic clock retardation predicted by Special Relativity

This model is intended to provide a more fundamental explanation for De Broglie's "meta law of Nature", via an analogy between matter waves and sound waves

A sound wave is a longitudinal wave that carries momentum. The quantity of momentum is a function of, among other things, wavelength. As a sound source moves, relative to its air medium, the "outgoing" waves in the forward direction are shortened by various amounts and those in the backward direction are lengthened by 
similar amounts due to the Doppler effect. So, all other things being equal, every direction has its own unique wavelength and its own incremental wave momentum in that direction. Whether a sound is emitted from a source which is stationary or moving, momentum is carried away and never returns to the source. An additional important characteristic of sound waves, described by Resnick and Halliday (1962, April), is that two similar sound waves in opposite directions, from stationary sources, create a longitudinal standing wave with nodes across which no net momentum is transferred.

Unlike an energy supplied sound source, if a vibrating particle is stationary or in uniform motion any momentum leaving it must return to the particle or its properties would change over time. Therefore it is reasonable to assume that the sum of all outgoing and incoming wave carried momentum should be zero, relative to the particle, whether that particle is stationary or in uniform motion. It will be shown, mathematically, that when we sum (i.e. integrate) all of the outgoing and incoming momentum, relative to a local preferred reference frame, we are able to predict a Total Internal Momentum, and a Net Forward Momentum. Similarly, a Total Internal Energy and a Kinetic Energy can be predicted.

The quantities and speeds predicted above are all defined relative to a local preferred reference frame. By specifying a local preferred reference frame we have selected the Lorentz-Poincare' viewpoint on the Special Relativity equations. In this viewpoint, if moving objects have a physical Lorentz contraction and there is a physical Lorentz change in the pulse rate of moving atomic clocks, then if two separated clocks are synchronized by a light signal whose speed is assumed (incorrectly) to be c, the clocks will be desynchronized by the exact amount required to make all future one-way light speeds (in either direction) appear to be c. Thus in his book Galison (2003) concludes his explanation of this physical de synchronization with the statement "Two phenomena that occurred in different places could appear to be simultaneous even though they were not." The Einstein viewpoint on simultaneity, which carries more weight in the physics community, could be one of the stumbling blocks preventing the unification of relativity and quantum mechanics. It is important to understand that with the Lorentz-Poincare' viewpoint the changes in measuring instruments are not reciprocal. The change in the pulse period of a moving electromagnetic (or light) clock, for example, is considered to be physical. The pulse period of a moving particle, in our proposed theory, is also considered to be physical but need not be the same as the electromagnetic pulse period. Positive results from an experiment proposed by Wagner (2015, June) would support the Lorentz-Poincare' viewpoint on simultaneity.

\section{Matter Wave Momentum in a specific direction}

As mentioned in the introduction, an analogy between sound waves and matter waves is used to define the periodic quantities required to compute the momentum carried by a plane longitudinal matter wave through a specified area. The variables and constants defined below, and measured in the Local Preferred Reference Frame, are required. Later in the paper, the specified area will be infinitesimal for each specified Doppler direction.

$$
\begin{aligned}
& \lambda=\text { Wave Length in a Specific Direction } \\
& A_{m}=\text { Maximum Matter Wave Amplitude } \\
& A=\text { Matter Wave Amplitude at a given position and time } \\
& \rho=\text { Matter Wave Density at a given position and time } \\
& \rho_{0}=\text { Matter Wave Density in the undisturbed Local Preferred Reference Frame } \\
& \rho^{\prime}=\rho-\rho_{0} \quad \text { (i.e. the density above or below the undisturbed density) } \\
& c_{0}=\text { Matter Wave Speed at } \rho_{0} \\
& x=\text { Distance in direction of matter wave motion } \\
& t=\text { Time } \\
& u=\text { Speed of the matter wave oscillation at a given position and time } \\
& \gamma=\text { An unknown constant for matter waves (for sound in air it is the specific heat ratio of air) }
\end{aligned}
$$

The periodic displacement equation tells how far an infinitesimal matter wave element is from its equilibrium position. For longitudinal waves, this displacement is along the direction of matter wave motion. The maximum displacement is $A_{m}$ and the instantaneous displacement is $A$ for a given $x$ and $t$. Sin and cos functions are used to represent all periodic functions. The displacement equation is then given by;

$$
A=A_{m} \cos \frac{2 \pi}{\lambda}\left(x-c_{0} t\right)
$$


Commenting on wave momentum, Charles Peskin (2010, August) says "The phenomenon of wave momentum is remarkable in several respects. First, it is not clear a priori that waves ought to have associated momentum", he then goes on "Even in the longitudinal case, since the wave motion is typically oscillatory, one would think that the average momentum density would be zero. How can there be net momentum in the direction of the wave?". He also says "Momentum is a concept normally associated with particles, and the fact that waves have momentum is perhaps the first hint, even at the classical level, of wave-particle duality". The answer provided by Peskin involves the density and oscillation speed equations below. Both these wave equations have the same cycle and phase. This means that when the density is at its maximum value the oscillation speed is at its maximum forward value, but when the density is at its minimum value the oscillation speed is at its maximum backward value. Momentum is density times incremental volume times velocity. So when integration is performed for a single wave, the sum of all the forward momentum elements is greater than the sum of all the backward momentum elements, resulting in a net forward momentum for the wave.

The displacement speed of a matter wave element is obtained by taking the derivative of equation (1) with respect to time. This results in the following equation.

$$
u=\left(\frac{A_{m} 2 \pi c_{0}}{\lambda}\right) \sin \frac{2 \pi}{\lambda}\left(x-c_{0} t\right)
$$

The density wave equation is given by;

$$
\rho^{\prime}=\left(\frac{\gamma \rho_{0} A_{m} 2 \pi}{\lambda}\right) \sin \frac{2 \pi}{\lambda}\left(x-c_{0} t\right)
$$

The longitudinal matter wave momentum carried by a single plane wave in a tiny tube in the direction of wave propagation, where the wave front is perpendicular to that direction and the direction is measured in the local preferred reference frame, is given by the following integral;

$$
\int_{0}^{\lambda}(\rho u a) d x
$$

where "a" is the area of the tube perpendicular to the wave direction. While we include area to get the total momentum in the tube, Peskin computes total momentum per unit area but his explanation of how we get from an integral with $\rho$ in it to an integral with $\rho^{\prime}$ is essentially as described below. The following equality is true because $\rho^{\prime}$ has equal density above and below $\rho_{0} . \int_{0}^{\lambda} \rho^{\prime} d x=\int_{0}^{\lambda}\left(\rho-\rho_{0}\right) d x=0$. Similarly $\int_{0}^{\lambda} u d x=0$. Since $\rho=\rho_{0}+\rho^{\prime}$ equation (4) can be written as, $\int_{0}^{\lambda}(\rho u a) d x=\int_{0}^{\lambda}\left(\rho_{0} u+\rho^{\prime} u\right) a d x=\rho_{0} a \int_{0}^{\lambda} u d x+$ $\int_{0}^{\lambda} \rho^{\prime}$ uadx. Substituting 0 for the first integral we have;

$$
\int_{0}^{\lambda}(\rho u a) d x=\int_{0}^{\lambda}\left(\rho^{\prime} u a\right) d x
$$

Pulling constants outside the integral, using equations (2) and (3) to substitute for $\mathrm{u}$ and $\rho^{\prime}$, and setting $t=0$ for a snapshot in time, the momentum, $p$, for a single wave is;

$$
p=\gamma \rho_{0} c_{0}\left(\frac{A_{m} 2 \pi}{\lambda}\right)^{2} a \int_{0}^{\lambda} \sin ^{2}\left(\frac{2 \pi x}{\lambda}\right) d x
$$

Finally, after integrating, the longitudinal matter wave momentum carried by a single plane wave in a tube normal to the wave direction is given by;

$$
p=\frac{2 \gamma \rho_{0} c_{0} A_{m}^{2} \pi^{2} a}{\lambda}
$$




\section{Total Internal Momentum}

The Doppler effect on incremental matter wave momentum can be derived using the diagram and definitions in Figure 1. If the area, $a$, in equation (7) is replaced with the infinitesimal area, $d a$, then the infinitesimal momentum, $d p$, in a direction normal to $d a$ is given by;

$$
d p=\frac{2 \gamma \rho_{0} c_{0} A_{m}^{2} \pi^{2} d a}{\lambda}
$$

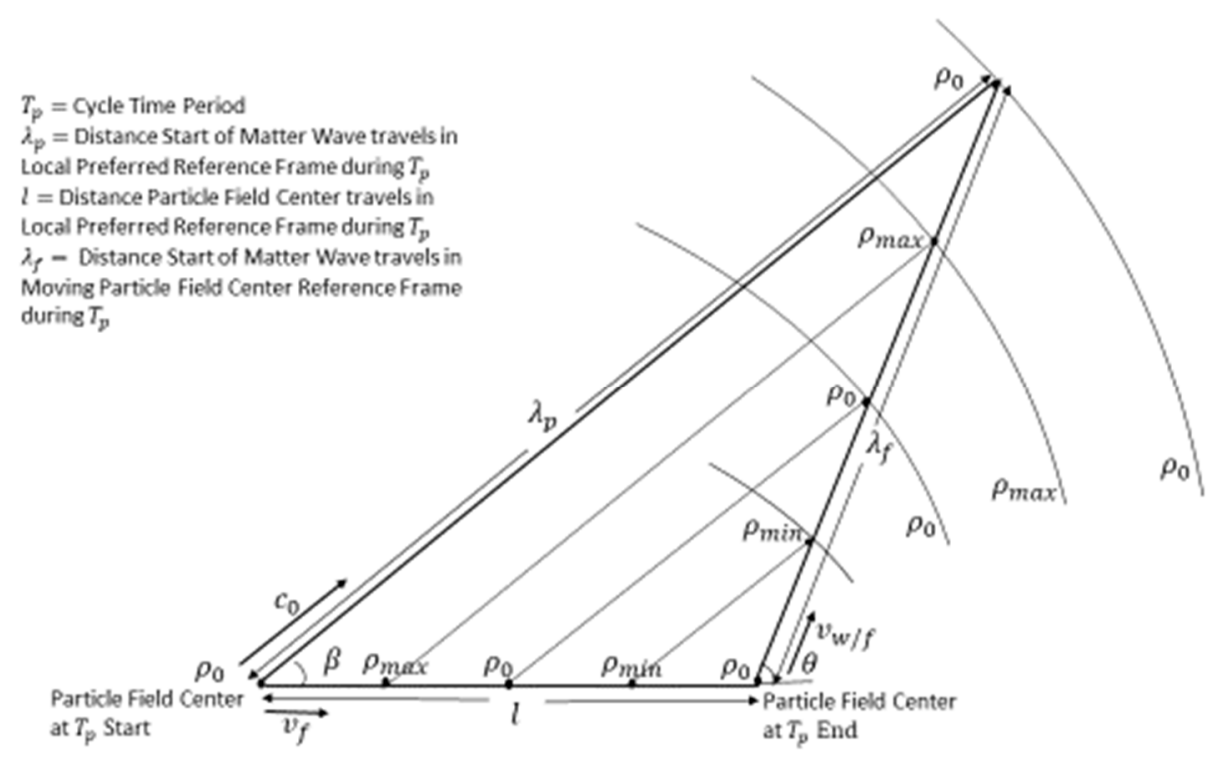

Figure 1. Variables for computing Matter Wave Momentum in $\beta$ direction

Selecting the angle $\beta$, between $\lambda_{p}$ and $l$ in Figure 1., $d p$ in the $\beta$ direction can be tracked for every phase of the wave. Key phases for wave density are shown by $\rho_{0}, \rho_{\max }, \rho_{0}, \rho_{\min }$, and $\rho_{0}$, in Figure1. Each of these phases reaches the $\lambda_{f}$ line simultaneously. The same is true for the wave displacement speed $u$ whose directions are all aligned with the $\beta$ direction. Thus as in section 2 . we have the two factors required to compute momentum, but this time the changing phases occur over $\lambda_{f}$ which is not aligned with the $u$ directions. We are, however, still able to compute the incremental momentum, as in section 2., but the integration must occur over $\lambda_{f}$ although the resulting sum is in the $\beta$ direction. Therefore, the $\lambda$ value in equation (8), for infinitesimal momentum delivered in the $\beta$ direction for one full matter wave, is $\lambda_{f}$ and we have;

$$
d p=\frac{2 \gamma \rho_{0} c_{0} A_{m}^{2} \pi^{2} d a}{\lambda_{f}}
$$

It can be seen from Figure 1. that $\lambda_{f}$ is a function of $\beta$ and therefore $d p$ has a Doppler influence that is a function of $\beta$. In Figure 1., $c_{0}$ is the matter wave velocity, and $v_{f}$ is the particle field center velocity, both relative to the local preferred reference frame. $v_{w / f}$ is the velocity of the matter wave increment relative to the moving particle field center reference frame. So, $\lambda_{p}=c_{0} T_{p}, \lambda_{f}=v_{w / f} T_{p}$, and $l=v_{f} T_{p}$.

In order to integrate with respect to $\beta$ we need $\lambda_{f}$ as a function of $\beta$. By the law of cosines;

$\lambda_{f}^{2}=\lambda_{p}^{2}+l^{2}-2 \lambda_{p} l \cos \beta$. Substituting using the equations above and solving for $\lambda_{f}$ gives;

$$
\lambda_{f}=T_{p} c_{0} \sqrt{1+\frac{v_{f}^{2}}{c_{0}^{2}}-2 \frac{v_{f}}{c_{0}} \cos \beta}
$$

Substituting $\lambda_{f}$ into equation (9) and eliminating $c_{0}$ in the numerator and denominator gives;

$$
d p=\frac{2 \gamma \rho_{0} A_{m}^{2} \pi^{2} d a}{T_{p} \sqrt{1+\frac{v_{f}^{2}}{c_{0}^{2}}-2 \frac{v_{f}}{c_{0}} \cos \beta}}
$$


Equation (11) is the infinitesimal single matter wave momentum in the $\beta$ direction at particle field center velocity $v_{f}$.

Figure 2. depicts the doppler shifted inward and outward waves. A description of the picture and related variables from Figure 1. is provided in Figure 2.

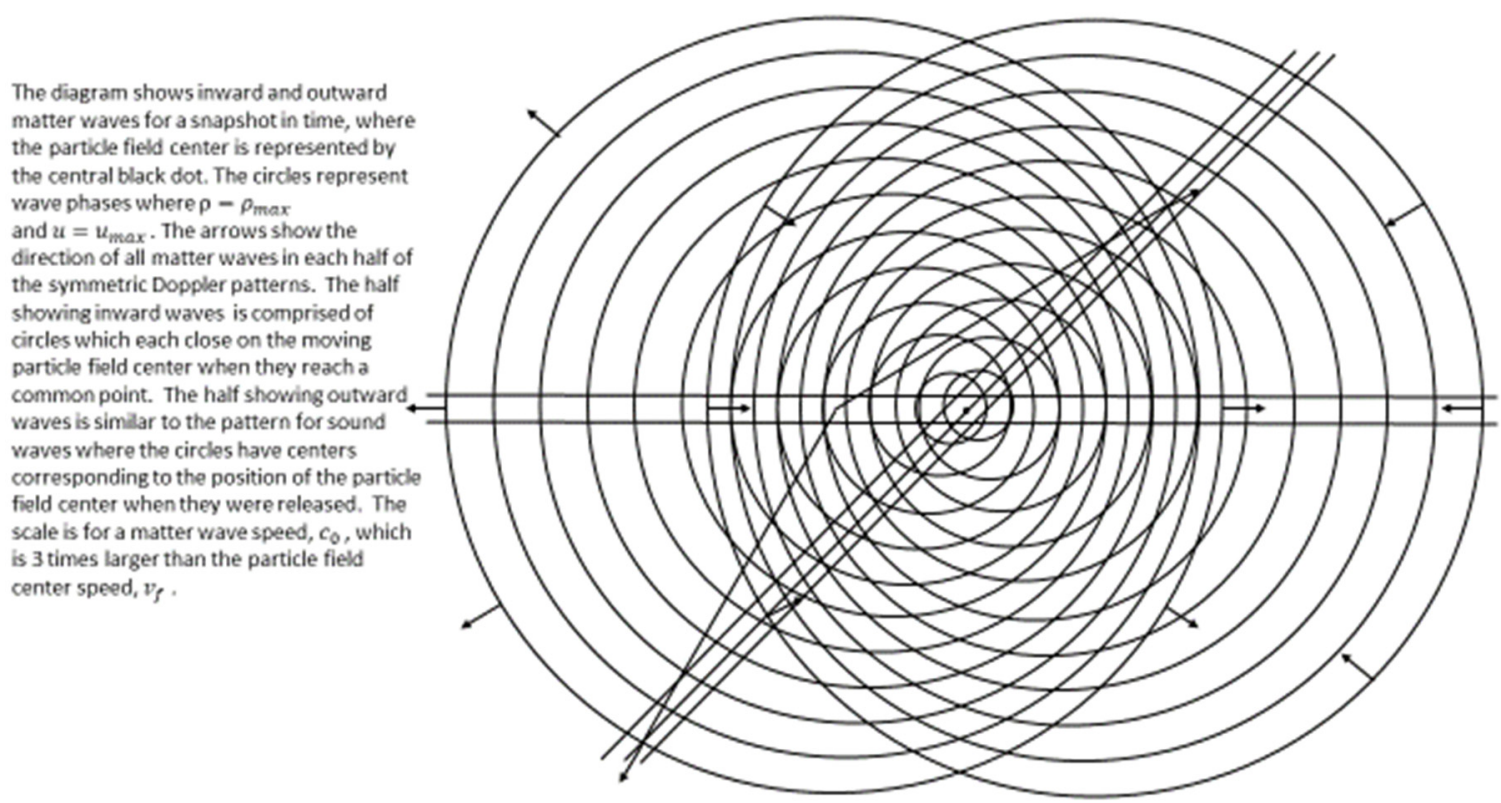

Figure 2. Doppler Inward and Outward Matter Waves

Two infinitesimal corridors passing through the moving particle field center are shown as examples of wave momentum, $d p$, traveling in opposite directions, in order to explain what is intended by integration of $d p$ in the $\beta$ direction.

The angles of the corridors shown in Figure 2. are sample values for the angle $\theta$ shown in Figure 1. Similarly, the angles of the little arrows are at sample values for the angle $\beta$ also shown in Figure 1. The easiest example to visualize is for waves in the horizontal corridor where $\theta=0$ and $\beta=0$. Note that the small inward waves moving to the right, on the left of the black dot, become the same size small outward waves, also moving to the right, on the right of the black dot, after passing through the moving particle field center. Also, the large inward waves moving to the left become the same size large outward waves, also moving to the left, after passing through the moving particle field center. In the first case incremental momentum moves "forward", while in the second case it moves "backward". When integrating, any angle $\beta$ represents all the incremental momentum traveling relative to the local preferred reference frame in the $\beta$ direction. This includes both the inward and outward components of that direction as described above.

A given phase of any single matter wave forms a circle about the point from which it was released because the wave speed is $c_{0}$ in any direction relative to the local preferred reference frame. Consider the mid-phase circle of radius $\mathrm{R}$ for one representative wave. The area $d a$ normal to the $\beta$ direction can be represented as $d a=$ $R d \beta(R d \alpha)$ where $R d \beta$ is in the plane formed by $\lambda_{p}$ and $l$, and $R d \alpha$ is perpendicular to that plane. Then by integrating from zero to $2 \pi$ with respect to $\beta$, the total unsigned momentum from all directions in that infinitesimally thin plane can be obtained. One additional integration is required to sum up all the planes in a representative shape. If a particle sphere model is used, all planes can be included by integrating with respect to $\alpha$ from zero to $\pi$. If a cylindrical model is used $R d \alpha$ can be replaced by $d z$, where $z$ is an axis perpendicular to the plane referred to above, and integration is from zero to an unknown cylinder length, $z$. We select $d z$, but in either case the related integration produces another unknown constant multiplier which ultimately results in one combined unknown multiplier, or constant of proportionality, which must be set empirically. So it doesn't matter which integration is selected.

The integral for $d z$ from 0 to $z$ is simply $z$ so both the $R$ from $R d \beta$ and the $z$ can be pulled outside the remaining required integral along with all other assumed constants, resulting in; 


$$
p=\frac{2 \gamma \rho_{0} A_{m}^{2} \pi^{2} R z}{T_{p}} \int_{\beta=0}^{\beta=2 \pi} \frac{d \beta}{\sqrt{1+\frac{v_{f}^{2}}{c_{0}^{2}}-2 \frac{v_{f}}{c_{0}} \cos \beta}}
$$

We assume that the wave amplitude, $A_{m}$, remains constant at a given $v_{f}$ value. At this point, we also tentatively assume that $\frac{A_{m}^{2}}{T_{p}}$ remains constant as $v_{f}$ changes. This is done to see how close the function parts of our Net Forward Momentum and Kinetic Energy match existing experimental results and Special Relativity Theory, when the only thing changing is doppler shifted wavelengths.

Since the integrals and constants are repeated in each of the momentum and energy quantities that will be determined, it is convenient to define the following;

$$
\begin{gathered}
I 1=\frac{\cos \beta d \beta}{\sqrt{1+\frac{v_{f}^{2}}{c_{0}^{2}}-2 \frac{v_{f}}{c_{0}} \cos \beta}} \\
I 2=\frac{d \beta}{\sqrt{1+\frac{v_{f}^{2}}{c_{0}^{2}}-2 \frac{v_{f}}{c_{0}} \cos \beta}} \\
Q_{M}=2 \gamma\left(\rho_{0} \pi^{2} R z\right) \\
Q_{E}=\left(\rho_{0} \pi^{2} R z\right) c_{0}
\end{gathered}
$$

This will allow easier comparison of the quantities and emphasize their differences. Equation (12), for example, is rewritten as;

$$
p=Q_{M}\left(\frac{A_{m}^{2}}{T_{p}}\right) \int_{\beta=0}^{\beta=2 \pi} I 2
$$

As will be seen later, the $I 2$ integral appears in three of the four momentum and energy quantities. We desire to compute the $I 2$ integral for a given value of $v_{f}$. After difficulty with a closed form or series solution, the approach taken was to select representative $v_{f}$ values in the range from zero to near $c_{0}$ and use the online Integral Calculator (2020) to provide an accurate integrated value for each selected $\frac{v_{f}}{c_{0}}$ value.

As will be shown later, the Total Internal Matter Wave Kinetic Energy, which will be used to compute the Kinetic Energy added to a particle, is functionally identical to the Total Internal Matter Wave Momentum, except for the constants $Q_{M}$ and $Q_{E}$.

\section{Net Forward Momentum}

As explained in section 3 , the angle $\beta$ specifies the direction of the incremental matter wave momentum relative to the local preferred reference frame. This includes the momentum from both inward and outward waves in the $\beta$ direction as described for Figure 2.

For a given $\beta$ angle, the component of the incremental matter wave momentum aligned with the $v_{f}$ direction is $d p \cos \beta$ where $d p$ is specified by equation (11). We define the Net Forward Momentum, $p_{f}$, as the sum of the $v_{f}$ aligned components of incremental matter wave momentum for all $\beta$ angles, where;

$$
p_{f}=\frac{2 \gamma \rho_{0} A_{m}^{2} \pi^{2} R z}{T_{p}} \int_{\beta=0}^{\beta=2 \pi} \frac{\cos \beta d \beta}{\sqrt{1+\frac{v_{f}^{2}}{c_{0}^{2}}-2 \frac{v_{f}}{c_{0}} \cos \beta}}
$$

Or in the shorthand notation presented in section 3;

$$
p_{f}=Q_{M}\left(\frac{A_{m}^{2}}{T_{p}}\right) \int_{\beta=0}^{\beta=2 \pi} I 1
$$

Momentum components perpendicular to $v_{f}$ (i.e. $d p \sin \beta$ ) are ignored because these components cancel one 
another. Every $\beta$ angle in the range 0 to $\pi$ has a symmetrical $\beta$ angle in the range $\pi$ to $2 \pi$ with the same incremental momentum magnitude. It should also be noted that $\beta$ angles from $-\frac{\pi}{2}$ to $+\frac{\pi}{2}$ have momentum components in the $+v_{f}$ direction while $\beta$ angles from $\frac{\pi}{2}$ to $\frac{3 \pi}{2}$ have momentum components in the $-v_{f}$ direction.

As mentioned earlier, we have tentatively assumed that $\frac{A_{m}^{2}}{T_{p}}$ remains constant as $v_{f}$ changes. If, however, it is not constant then it can be represented as;

$$
\frac{A_{m}^{2}}{T_{p}}=\frac{A_{m 0}^{2}}{T_{0}} U
$$

where $A_{m 0}$ and $T_{0}$ are the values of $A_{m}$ and $T_{p}$ respectively when a particle is at rest relative to the local preferred reference frame, and $U$ is an unknown function of $v_{f}$. We now define a single constant of proportionality;

$$
K_{M}=Q_{M}\left(\frac{A_{m 0}^{2}}{T_{0}}\right)
$$

such that our Net Forward Momentum is:

$$
p_{f}=K_{M} U \int_{\beta=0}^{\beta=2 \pi} I 1
$$

The approach for finding the value of $K_{M}$ is to approximate the variable part of equation (22), $U \int_{\beta=0}^{\beta=2 \pi} I 1$, for $v_{f} \ll c_{0}$ and set the result of equation (22) equal to the classical momentum at $v_{f}$ since we know that our forward momentum prediction must approximate the classical value at low values of $v_{f}$. By use of the Binomial Theorem $\frac{I 1}{\cos \beta d \beta}$ (see eq (13)) can be approximated by;

$$
\frac{I 1}{\cos \beta d \beta} \approx 1+\frac{v_{f}}{c_{0}} \cos \beta-\frac{1}{2} \frac{v_{f}^{2}}{c_{0}^{2}}+\frac{3}{2} \frac{v_{f}^{2}}{c_{0}^{2}} \cos ^{2} \beta-\frac{3}{2} \frac{v_{f}^{3}}{c_{0}^{3}} \cos \beta+\frac{3}{8} \frac{v_{f}^{4}}{c_{0}^{4}}
$$

A low speed approximation for $\int_{\beta=0}^{\beta=2 \pi} I 1$ can then be obtained by multiplying each of the 6 terms in equation (23) by $\cos \beta d \beta$ and integrating each term. The result is;

$$
\int_{\beta=0}^{\beta=2 \pi} I 1 \approx \frac{\pi v_{f}}{c_{0}}-\frac{3 \pi}{2} \frac{v_{f}^{3}}{c_{0}^{3}} \approx \frac{\pi v_{f}}{c_{0}}
$$

Next we must approximate $U$ for $v_{f} \ll c_{0}$. The process for obtaining $U\left(v_{f}\right)$ will be explained in the section on Kinetic Energy. The function determined there is;

$$
U=\frac{1}{\left(1-\frac{v_{f}^{2}}{c_{0}^{2}}\right)^{1 / 4}}
$$

Again, using the Binomial Theorem we have, for $v_{f} \ll c_{0} ; U \approx 1+\frac{1}{4}\left(\frac{v_{f}^{2}}{c_{0}^{2}}\right)+\left(\frac{\left(\frac{1}{4}\right)^{2}+\frac{1}{4}}{2}\right)\left(\frac{v_{f}^{4}}{c_{0}^{4}}\right)$. Neglecting the 
high order term, we have; $U \approx 1+\frac{1}{4}\left(\frac{v_{f}^{2}}{c_{0}^{2}}\right)$ so $U \int_{\beta=0}^{\beta=2 \pi} I 1 \approx\left[1+\frac{1}{4}\left(\frac{v_{f}^{2}}{c_{0}^{2}}\right)\right]\left[\pi \frac{v_{f}}{c_{0}}\right]$. Multiplying and again eliminating the high order term gives;

$$
p_{f}=K_{M}\left(\frac{\pi v_{f}}{c_{0}}\right)
$$

The classical value for momentum at $v_{f}$ is $m_{0} v_{f}$. Setting this equal to equation (26) and solving for $K_{M}$ gives finally;

$$
K_{M}=\frac{m_{0} c_{0}}{\pi}
$$

An EXCEL program was written to compute results for 18 selected input values of $\frac{v_{f}}{c_{0}}$ in the range $0<v_{f}<c_{0}$. The calculations were made for an electron where $m_{0}=9.109534 \times 10^{-31} \mathrm{~kg}$ and $c_{0}=299,792,458.0 \mathrm{~m} / \mathrm{s}$ resulting in $K_{M}=8.69294619 \times 10^{-23} \mathrm{~kg}(\mathrm{~m} / \mathrm{s})$. The online Integral Calculator (2020) was used to obtain the "I1 integral" for each $\frac{v_{f}}{c_{0}}$ value and these values were also input to the EXCEL program. In addition to computing values for $p_{f}$, comparative values for the Relativistic Momentum, $p_{r}$, and the Classical Momentum, $p_{c}$, were also computed. Considerable testing and sanity checking were performed to ensure the accuracy of the results.

Table 1 shows predictions for 7 representative values of $\frac{v_{f}}{c_{0}}$ prior to introducing the $U$ function, given by equation (25), which provides the best balance between experimental results for both Forward Momentum and Kinetic Energy. Values are rounded for ease of comparison.

Table 1. Net Forward Momentum (Doppler effect only) - in $\mathrm{kg}(\mathrm{m} / \mathrm{s})$

\begin{tabular}{cccccc}
\hline$\frac{v_{f}}{c_{0}}$ & $\int_{\beta=0}^{\beta=2 \pi} I 1$ & $p_{f}$ & $p_{r}$ & $\frac{p_{f}}{p}$ \\
& & & & $p_{c}$ & \\
$(\%$ of Total Mom. $)$ \\
\hline 0.01 & 0.031417102 & $2.73 \times 10^{-24}$ & $2.73 \times 10^{-24}$ & $2.73 \times 10^{-24}$ & 0.5 \\
0.3 & 0.976202066 & $8.49 \times 10^{-23}$ & $8.59 \times 10^{-23}$ & $8.19 \times 10^{-23}$ & 25.9 \\
0.5 & 1.74630563 & $1.52 \times 10^{-22}$ & $1.58 \times 10^{-22}$ & $1.37 \times 10^{-22}$ & 37.9 \\
0.7 & 2.80018000 & $2.43 \times 10^{-22}$ & $2.68 \times 10^{-22}$ & $1.91 \times 10^{-22}$ & $2.18 \times 10^{-22}$ \\
0.8 & 3.59480000 & $3.12 \times 10^{-22}$ & $3.64 \times 10^{-22}$ & $2.54 \times 10^{-22}$ & 62.1 \\
0.96 & 5.61350000 & $4.88 \times 10^{-22}$ & $6.91 \times 10^{-22}$ & $2.62 \times 10^{-22}$ & \\
\hline
\end{tabular}

From equations (22), (25), and (13), when $v_{f}=0, p_{f}=K_{M} \int_{\beta=0}^{\beta=2 \pi} \cos \beta d \beta=0$, but from equation (10) $\lambda_{f}=$ $T_{p} c_{0}$ at $v_{f}=0$. The rest value of $T_{p}$ is $T_{0}$ so the rest wavelength is $\lambda_{f 0}=T_{0} c_{0}$. Thus our wavelength related to momentum is not required to be infinite when the forward momentum is zero, as it is in the DeBroglie relationship $\lambda=h / p$.

From equations (19) and (17) $\frac{p_{f}}{p}=\frac{\int_{\beta=0}^{\beta=2 \pi} I 1}{\int_{\beta=0}^{\beta=2 \pi} I 2}$ regardless of whether or not $\frac{A_{m}^{2}}{T_{p}}$ is a function of $v_{f}$. Using values 
of $\int_{\beta=0}^{\beta=2 \pi} I 1$ from Table 1 and $\int_{\beta=0}^{\beta=2 \pi} I 2$ from the Kinetic Energy Table 3, we can compute the percentage of Total Internal Momentum that is carried as Net Forward Momentum relative to the local preferred reference frame. From the $\frac{p_{f}}{p}$ column in Table 1, notice how percentage rises from $0.5 \%$ at $\frac{v_{f}}{c_{0}}=0.01$ to $62.1 \%$ at $\frac{v_{f}}{c_{0}}=$ 0.96 . This shows that due only to the Doppler effect on the wavelengths of forward and backward matter waves, more and more momentum is carried in the forward waves as velocity increases.

Comparing the three momentum predictions in Table 1 it can be seen that they differ as follows;

At the lowest value for $\frac{v_{f}}{c_{0}}$ the three values are the same, as they should be. At a value of 0.3 (i.e. $30 \%$ of the speed of light) both $p_{f}$ and $p_{r}$ are larger than the classical value of $p_{c}$, but the relativistic value, $p_{r}$, is larger than the Net Forward Momentum value, $p_{f}$. This trend continues all the way through $\frac{v_{f}}{c_{0}}=0.96$. Changing the value of $K_{M}$ to a different constant value will not bring the $p_{f}$ predictions into better agreement with the relativistic predictions and related experimental values. When the $U$ function given by equation (25) is introduced, however, the better $p_{f}$ predictions in Table 2 are produced.

Equation (22) was used to compute the 18 predictions presented as curves in Figure 3. and to compute the 7 predictions selected for Table 2 below.

Table 2. Net Forward Momentum (with $U$ function) - in $\mathrm{kg}(\mathrm{m} / \mathrm{s})$

\begin{tabular}{cccc}
\hline$\frac{v_{f}}{c_{0}}$ & $p_{f}$ & $p_{r}$ & $p_{c}$ \\
\hline 0.01 & $2.73 \times 10^{-24}$ & $2.73 \times 10^{-24}$ & $2.73 \times 10^{-24}$ \\
0.3 & $8.69 \times 10^{-23}$ & $8.59 \times 10^{-23}$ & $8.19 \times 10^{-23}$ \\
0.5 & $1.63 \times 10^{-22}$ & $1.58 \times 10^{-22}$ & $1.37 \times 10^{-22}$ \\
0.7 & $2.88 \times 10^{-22}$ & $2.68 \times 10^{-22}$ & $1.91 \times 10^{-22}$ \\
0.8 & $4.03 \times 10^{-22}$ & $3.64 \times 10^{-22}$ & $2.18 \times 10^{-22}$ \\
0.93 & $8.05 \times 10^{-22}$ & $6.91 \times 10^{-22}$ & $2.54 \times 10^{-22}$ \\
0.96 & $11.0 \times 10^{-22}$ & $9.36 \times 10^{-22}$ & $2.62 \times 10^{-22}$ \\
\hline
\end{tabular}

Whereas in Table 1. our matter wave predictions for forward momentum are lower than the relativistic predictions, in Table 2 they are higher. Experimental results from a paper by Rogers (1940, March) were translated into momentum vs $\frac{v_{f}}{c_{0}}$ to allow comparison against our matter wave predictions and relativistic predictions. The $3 \frac{v_{f}}{c_{0}}$ values in the Rogers experiment center around our table value of 0.7 . For 2 of the 3 values, results from the experiment are higher than the relativity predictions, but not as high as ours. Error boxes in the Rogers paper translate to a positive error of roughly $0.02 \times 10^{-22} \mathrm{~kg}(\mathrm{~m} / \mathrm{s})$ but this still does not allow predictions as high as our matter wave predictions. The curves in Figure 3. Show the difference in predictions for matter wave, relativistic, and classical momentum. 


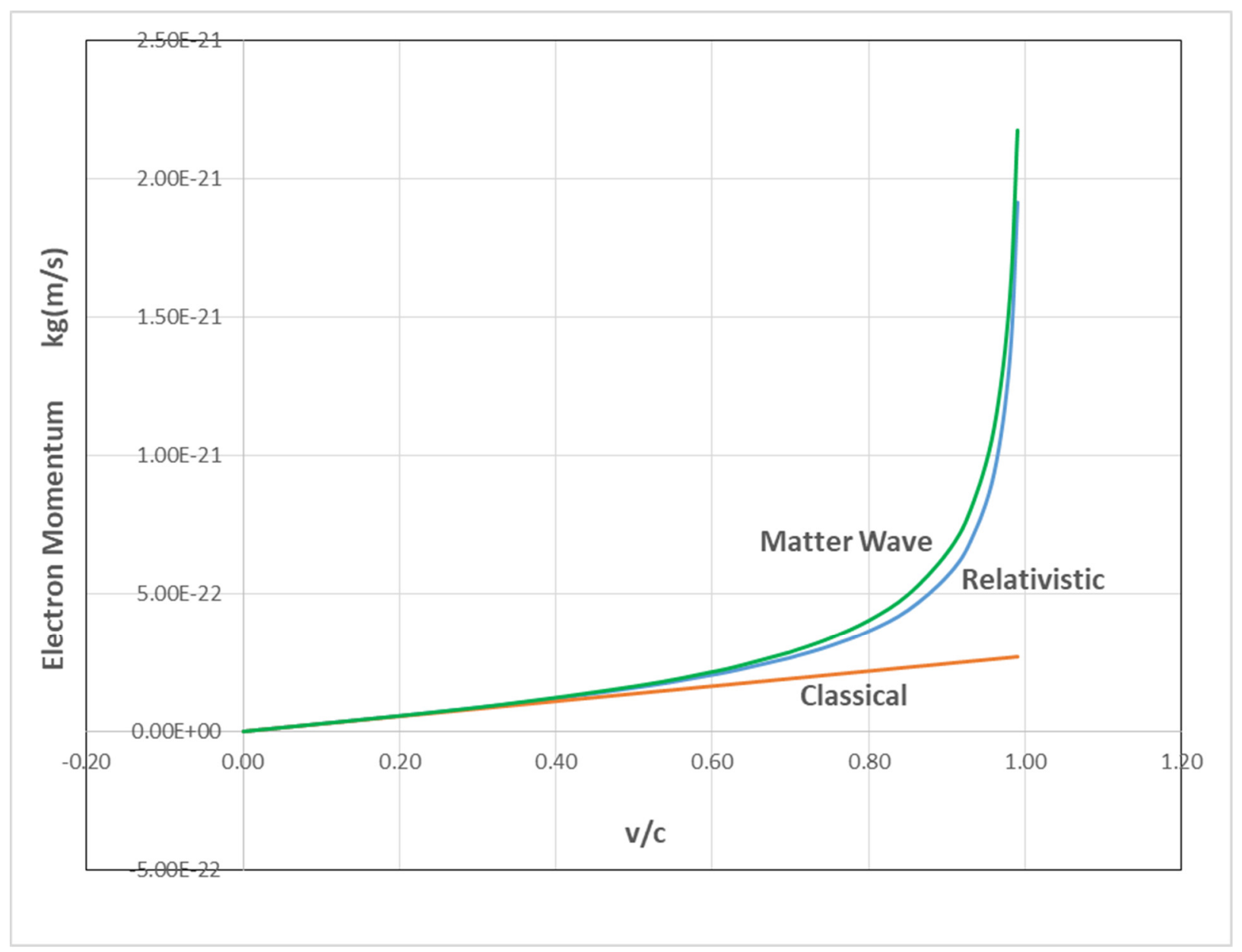

Figure 3. Momentum Predictions (with $U$ function for Matter Wave)

\section{Kinetic Energy}

Paralleling section 2 on matter wave momentum in a specific direction, the analogy between sound waves and matter waves is continued to compute the kinetic energy carried by a plane longitudinal matter wave through a specified area. Kinetic energy is $1 / 2$ density times incremental volume times velocity squared, so similar to equation (4), for momentum, the longitudinal matter wave kinetic energy carried by a single plane wave, in the direction of wave propagation relative to the local preferred reference frame, is given by;

$$
\int_{0}^{\lambda} \frac{1}{2}\left(\rho u^{2} a\right) d x
$$

Recalling that $\rho=\rho_{0}+\rho^{\prime}$ gives;

$$
\int_{0}^{\lambda} \frac{1}{2}\left(\rho u^{2} a\right) d x=\frac{1}{2} \rho_{0} a \int_{0}^{\lambda} u^{2} d x+\frac{1}{2} a \int_{0}^{\lambda} \rho^{\prime} u^{2} d x
$$

Substituting with equations (2) and (3) and setting $t=0$ for a snapshot in time, the kinetic energy for a single wave is;

$$
E=\frac{1}{2} \rho_{0} c_{0}^{2}\left(\frac{A_{m} 2 \pi}{\lambda}\right)^{2} a \int_{0}^{\lambda} \sin ^{2}\left(\frac{2 \pi x}{\lambda}\right) d x+\frac{1}{2} \gamma \rho_{0} c_{0}^{2}\left(\frac{A_{m} 2 \pi}{\lambda}\right)^{3} a \int_{0}^{\lambda} \sin ^{3}\left(\frac{2 \pi x}{\lambda}\right) d x
$$

The $2^{\text {nd }}$ integral in equation (30) gives zero, and the $1^{\text {st }}$ integral is the same as that in equation (6). After 
integrating, the longitudinal matter wave kinetic energy carried by a single plane wave is;

$$
E=\frac{\rho_{0} c_{0}^{2} A_{m}^{2} \pi^{2} a}{\lambda}
$$

where equation (31) is similar to equation (7). Note that $\gamma$ which is in momentum equation (7), has disappeared from kinetic energy equation (31) because the $2^{\text {nd }}$ integral in equation (30) is zero. Using the same reasoning as in section 3, the infinitesimal kinetic energy delivered in the $\beta$ direction for one full matter wave is;

$$
d E=\frac{\rho_{0} c_{0}^{2} A_{m}^{2} \pi^{2} d a}{\lambda_{f}}
$$

where equation (32) is similar to equation (9) and $\lambda_{f}$ is given by equation (10). Continuing with the reasoning in section 3 , we arrive at

$$
E=\frac{\rho_{0} c_{0} A_{m}^{2} \pi^{2} R z}{T_{p}} \int_{\beta=0}^{\beta=2 \pi} \frac{d \beta}{\sqrt{1+\frac{v_{f}^{2}}{c_{0}^{2}}-2 \frac{v_{f}}{c_{0}} \cos \beta}}
$$

where equation (33) is similar to equation (12). Using the shorthand notation of equations (14) and (16) gives;

$$
E=Q_{E}\left(\frac{A_{m}^{2}}{T_{p}}\right) \int_{\beta=0}^{\beta=2 \pi} I 2
$$

where equation (34) is similar to equation (17) and gives the Total Internal Matter Wave Kinetic Energy relative to the local preferred reference frame. This energy at rest is given by equation (34) when $v_{f}=0$.The integral, shown in full in equation (33), becomes $\int_{\beta=0}^{\beta=2 \pi} d \beta=2 \pi$, when $v_{f}=0$. Also, $U=1$ for $v_{f}=0$ which means that $\frac{A_{m}^{2}}{T_{p}}=\frac{A_{m 0}^{2}}{T_{0}}$ and therefore the rest energy is;

$$
E_{0}=Q_{E}\left(\frac{A_{m 0}^{2}}{T_{0}}\right)(2 \pi)
$$

The Matter Wave Kinetic Energy is defined as the kinetic energy added to a particle at rest in the local preferred reference frame in order to reach $v_{f}$, that is to say, the increase in Total Internal Matter Wave Kinetic Energy from the "rest" state. As such it is given by;

$$
E_{K}=Q_{E}\left(\frac{A_{m 0}^{2}}{T_{0}}\right) U \int_{\beta=0}^{\beta=2 \pi} I 2-Q_{E}\left(\frac{A_{m 0}^{2}}{T_{0}}\right)(2 \pi)
$$

As in section 4, we now define a single constant of proportionality;

$$
K_{K}=Q_{E}\left(\frac{A_{m 0}^{2}}{T_{0}}\right)
$$

The Matter Wave Kinetic Energy can then be written as;

$$
E_{K}=K_{K}\left[U \int_{\beta=0}^{\beta=2 \pi} I 2-(2 \pi)\right]
$$

The approach for finding the value of $K_{K}$ is to approximate the variable part of equation (38), $U \int_{\beta=0}^{\beta=2 \pi} I 2$, for $v_{f} \ll c_{0}$ and set the result of equation (38) equal to the classical kinetic energy at $v_{f}$ since we know that our kinetic energy prediction must approximate the classical value at low values of $v_{f}$. We already have the 
approximate value of the $U$ function given in equation (25); $U \approx 1+\frac{1}{4}\left(\frac{v_{f}^{2}}{c_{0}^{2}}\right)$. A procedure using the Binomial Theorem, and similar to that outlined in section 4 to arrive at equation (24), was used to obtain;

$$
\int_{\beta=0}^{\beta=2 \pi} I 2 \approx 2 \pi+\frac{\pi}{2} \frac{v_{f}^{2}}{c_{0}^{2}}+\frac{3 \pi}{4} \frac{v_{f}^{4}}{c_{0}^{4}} \approx 2 \pi+\frac{\pi}{2} \frac{v_{f}^{2}}{c_{0}^{2}}
$$

neglecting the high order term. Substituting the low $v_{f}$ approximations for $U$ and $\int_{\beta=0}^{\beta=2 \pi} I 2$ into equation (38) and neglecting the high order term, we have;

$$
E_{K} \approx K_{K}\left(\frac{\pi v_{f}^{2}}{c_{0}^{2}}\right)
$$

The classical value for kinetic energy at $v_{f}$ is $\frac{1}{2} m_{0} v_{f}^{2}$. Setting this equal to equation (40) and solving for $K_{K}$ gives finally;

$$
K_{K}=\frac{m_{0} c_{0}^{2}}{2 \pi}
$$

From equations (35) and (37);

$$
E_{0} \approx K_{K}(2 \pi)=m_{0} c_{0}^{2}
$$

Equation (42) is the relativistic rest energy and this is one of the reasons why equation (25) is a good choice for the $U$ function.

From equations (21), (15), and (27) the rest mass, $m_{0}$, can be expressed as $m_{0}=\frac{2 \gamma\left(\rho_{0} \pi^{3} R z\right)}{c_{0}}\left(\frac{A_{m 0}^{2}}{T_{0}}\right)$. But from equations (37), (16), and (41) the rest mass can also be expressed as $m_{0}=\frac{2\left(\rho_{0} \pi^{3} R z\right)}{c_{0}}\left(\frac{A_{m 0}^{2}}{T_{0}}\right)$. There is, of course, one and only one value for the rest mass, so the ratio of specific heats must be;

$$
\gamma=1.0
$$

Up to this point we have been referring to the matter wave bearing medium in our sound wave analogy as a gas, namely air. It is hard to justify $\gamma=1.0$ for a gas, but note that for, say, water at $40 \operatorname{deg} \mathrm{F} \gamma=1.000$ to 3 decimal places. So if the viewpoints in this paper are a close representation of physical reality, we are able to conclude that the medium which bares longitudinal matter waves behaves more like a liquid than a gas. i e. It is not very compressible.

An EXCEL program was written to compute results for 18 selected input values in the range $0<v_{f}<c_{0}$ The value of $K_{K}$ for an electron, as given by equation (41), is $K_{K}=1.30303985 \times 10^{-14} \mathrm{~kg}(\mathrm{~m} / \mathrm{s})^{2}$. The online Integral Calculator (2020) was used to obtain the "I2 integral" for each $\frac{v_{f}}{c_{0}}$ value, and these values were also input to the EXCEL program. Values for the $U$ function were computed using equation (25) i.e. the same function used to compute Net Forward Momentum for Table 2 and Figure 3. In addition to computing values for $E_{K}$, comparative values for the Relativistic Kinetic Energy, $E_{K r}$, and the Classical Kinetic Energy, $E_{K c}$, were also computed.

Table 3 shows predictions for 7 representative values of $\frac{v_{f}}{c_{0}}$. Kinetic Energy values are rounded for ease of 
comparison.

Table 3. Kinetic Energy (with $U$ function) - in $\mathrm{kg}(\mathrm{m} / \mathrm{s})^{2}$

\begin{tabular}{ccccc}
\hline$\frac{v_{f}}{c_{0}}$ & $\int_{\beta=0}^{\beta=2 \pi} I 2$ & $E_{K}$ & $E_{K r}$ & $E_{K c}$ \\
\hline 0.01 & 6.283342396 & $4.09 \times 10^{-18}$ & $4.09 \times 10^{-18}$ & $4.09 \times 10^{-18}$ \\
0.5 & 6.743001419 & $1.25 \times 10^{-14}$ & $1.27 \times 10^{-14}$ & $1.02 \times 10^{-14}$ \\
0.7 & 7.382800 & $3.20 \times 10^{-14}$ & $3.28 \times 10^{-14}$ & $2.01 \times 10^{-14}$ \\
0.8 & 7.981211111 & $5.24 \times 10^{-14}$ & $5.46 \times 10^{-14}$ & $2.62 \times 10^{-14}$ \\
0.91 & 9.305300 & $1.06 \times 10^{-13}$ & $1.16 \times 10^{-13}$ & $0.34 \times 10^{-13}$ \\
0.96 & 10.772600 & $1.83 \times 10^{-13}$ & $2.11 \times 10^{-13}$ & $0.38 \times 10^{-13}$ \\
0.99 & 13.426400 & $3.84 \times 10^{-13}$ & $4.99 \times 10^{-13}$ & $0.40 \times 10^{-13}$ \\
\hline
\end{tabular}

Figure 4 shows plots for the 18 selected $\frac{v_{f}}{c_{0}}$ values.

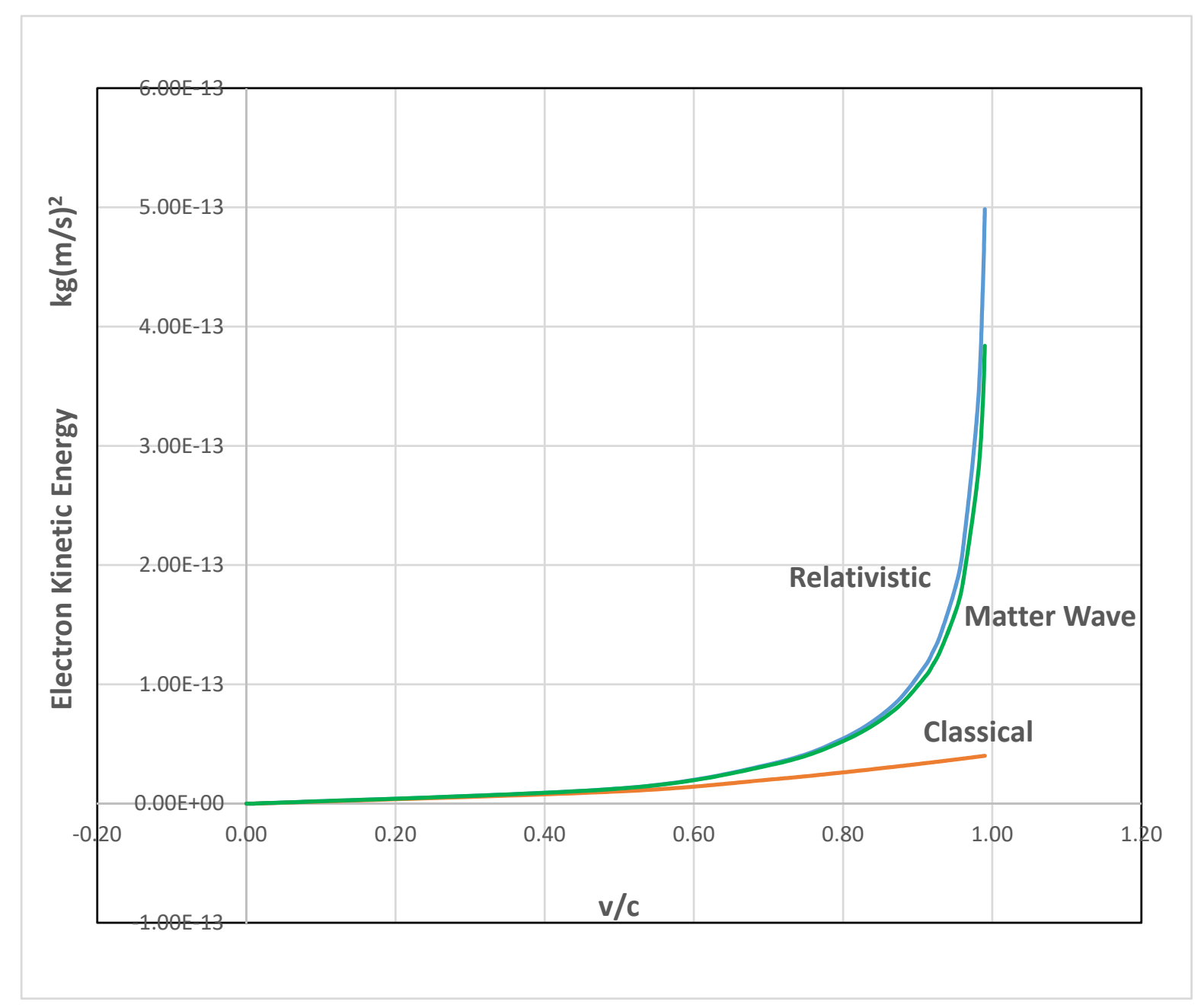

Figure 4. Kinetic Energy Predictions (with $U$ function for Matter Wave)

Looking at Table 3 and at the related plots in Figure 4, it can be seen that whereas our predictions for Net Forward Momentum were slightly higher than the Relativistic predictions, for Kinetic Energy they are slightly 
lower. Thus the $U$ function given by equation (25) provides a good balance if we are trying to match the very successful relativity predictions, and provides yet another reason for selecting that function. Despite the great success of the relativistic predictions, it should be noted that when Bertozzi's (1964) experimental results for kinetic energy are translated to allow comparison with our matter wave predictions, the average absolute difference between experimental value and predicted value, for Bertozzi's four $v_{f}$ values is $1.25 \times 10^{-13}$ $\mathrm{kg}(\mathrm{m} / \mathrm{s})^{2}$ for matter waves and $0.92 \times 10^{-13} \mathrm{~kg}(\mathrm{~m} / \mathrm{s})^{2}$ for relativity. Bertozzi's $\frac{v_{f}}{c_{0}}$ values for the experiment were $0.867,0.910,0.960$, and 0.987 , He obtained results for these four conditions using an accelerating potential difference. He then also obtained results for the last two $\frac{v_{f}}{c_{0}}$ values using thermal measurements. The thermal results show differences even further from the relativistic predictions, and also further from our predictions. But the point is that perhaps additional direct measurement momentum and kinetic energy experiments should be performed at relativistic speeds.

\section{Gravity}

A proper analysis of gravity, under the viewpoint taken in this paper, is beyond the scope of this paper. It is, however, worth considering how an extended analysis under this viewpoint might proceed because it brings to light an underlying assumption; that because a particle is considered to be nothing more than the sum of "in" and "out" matter waves focused through its center, whatever happens to these matter waves determines the future location of that center. i.e. a change in speed, or distortion though tiny slits.

Figure 5 shows a particle accelerating in earth's gravitational field, where $g$ is the local acceleration, and $v_{f}$ is the instantaneous velocity at the particle's position. The particle drops a distance, $d$, in one cycle time period, $T_{p}$. In this simplified analysis, it is assumed that the local preferred reference frame is essentially a non-rotating earth centered reference frame and that all distances and velocities are relative to that reference frame. As such $v_{f}$ is the same $v_{f}$ shown in Figure 1 used to compute $\lambda_{f}$ in equation (10). $S$ is the distance from the current particle position and is parallel to the velocity and acceleration. $c_{0}$ is the matter wave speed at $S=0$, and, $c_{G R}$, is the gradient of that speed at that position. For small distances away from $S=0$ the matter wave speed is given by $c=c_{0}+c_{G R} S$.

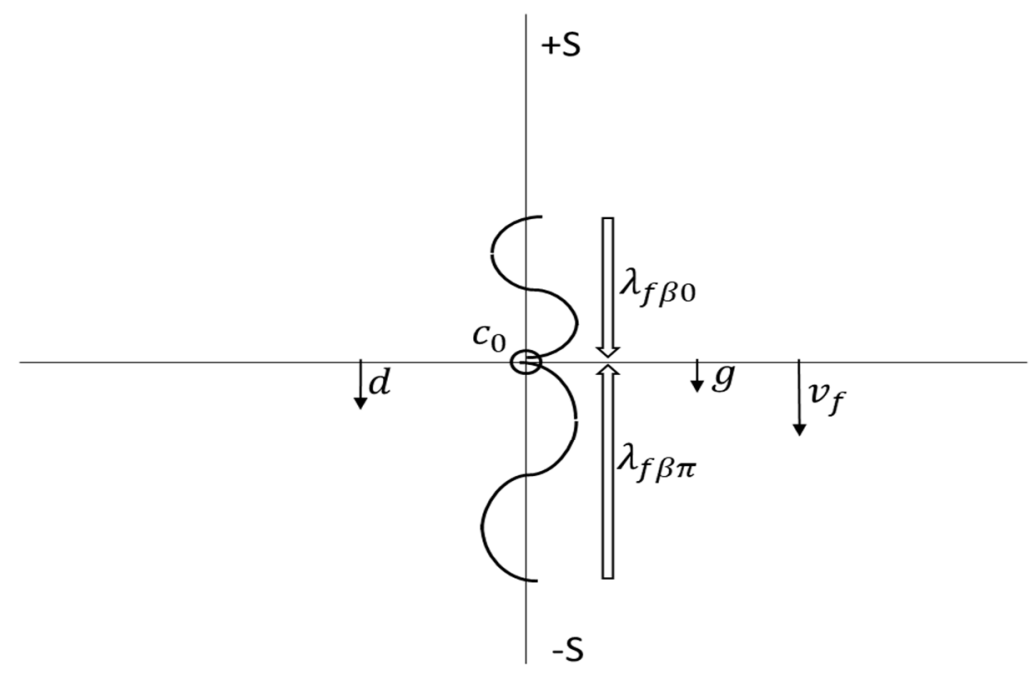

Earth

Figure 5. Gradient in Matter Wave Speed as cause of Gravity 
If $c_{G R}$ and $g$ were both equal to zero at the initial particle position shown, then equation (10) could be used to find the "forward" wavelength at $\beta=0$ as, $\lambda_{f \beta 0}=\left(c_{0}-v_{f}\right) T_{p}$, and the "backward" wavelength at $\beta=\pi$ as, $\lambda_{f \beta \pi}=\left(c_{0}+v_{f}\right) T_{p}$. The difference in these wavelengths would then be $\lambda_{f \beta \pi}-\lambda_{f \beta 0}=2 v_{f} T_{p}$, and the matter wave doppler shifted patterns would be similar to those shown in Figure 2 rotated 90 degrees clockwise. At higher values of $v_{f}$, as a particle falls, the difference in backward and forward wavelengths would be larger and the related doppler patterns more pronounced.

Our limited goal is to see how the difference in backward and forward wavelengths would need to change along the $\beta=0$ corridor when the particle drops a distance, $d$ during one $T_{p}$ cycle and to relate this to the light speed gradient given by General Relativity Theory. The tacit assumption that oppositely directed matter waves represent the components of harmonically oscillating pseudo standing waves is maintained for the accelerated case under consideration. Therefore these waves are still considered to pass through the particle center in the same duration of time, $T_{p}$. Referring to Figure 5 and the definitions provided above, the infinitesimal distance traveled by a matter wave is $d S=\left(c_{0}+C_{G R} S\right) d T_{p}$. To find the distance traveled by, say, the trailing edge of a a matter wave during the cycle time, $T_{p}$, the following integrations must be performed;

$$
\int d T_{p}=\int \frac{d S}{c_{0}+c_{G R} S}
$$

The indefinite integrals are given by;

$$
T_{p}=\frac{\ln \left(c_{G R} S+c_{0}\right)}{c_{G R}}+\text { constant }
$$

During the cycle time period the trailing edge of $\lambda_{f \beta 0}$ must travel from $S=\lambda_{f \beta 0}$ to $S=-d$. The solution with these boundary conditions is;

$$
T_{p}=\frac{\ln \left(c_{G R} \lambda_{f \beta 0}+c_{0}\right)}{c_{G R}}-\frac{\ln \left(-c_{G R} d+c_{0}\right)}{c_{G R}}
$$

Similarly, the trailing edge of $\lambda_{f \beta \pi}$ must travel from $S=-\lambda_{f \beta \pi}$ to $S=-d$ in the same positive cycle time, $T_{p}$. The solution with these boundary conditions is;

$$
T_{p}=\frac{\ln \left(-c_{G R} d+c_{0}\right)}{c_{G R}}-\frac{\ln \left(-c_{G R} \lambda_{f \beta \pi}+c_{0}\right)}{c_{G R}}
$$

Note that both wave trailing edges wind up at $S=-d$ at the same instant in time. Using the math identities $\ln \frac{x}{a}=\ln x-\ln a$ and $y=e^{x}$ if and only if $x=\ln y$ plus a bit of math and elimination of high order terms, we arrive at the following approximation;

$$
\lambda_{f \beta \pi}-\lambda_{f \beta 0} \approx 2 d+c_{G R} T_{p} d
$$

We know that $d=v_{f} T_{p}+\frac{1}{2} g T_{p}^{2}$. If the demonstrated bending of starlight past the sun, predicted by the final version of General Relativity, is correct, then the implication is that the $c_{0}$ gradient near a massive body is $c_{G R}=\frac{2 g}{c_{0}}$. If this is assumed to be the matter wave speed gradient near earth then substituting $d$ and $c_{G R}$ into equation (48) and eliminating high order terms gives;

$$
\lambda_{f \beta \pi}-\lambda_{f \beta 0} \approx 2 v_{f} T_{p}+\frac{2 v_{f} g T_{p}^{2}}{c_{0}}+g T_{p}^{2}
$$


Using the required wavelength difference, $2 v_{f} T_{p}$, mentioned earlier for the constant velocity case with no matter wave gradient or gravitational acceleration, we see that equation (49) requires a wavelength difference that is greater by $g T_{p}^{2}\left(1+\frac{2 v_{f}}{c_{0}}\right)$. It is interesting to note that for $v_{f}=0$, the additional wavelength difference is just twice the free fall distance of $\frac{1}{2} g T_{p}^{2}$.

\section{Conclusion}

Analysis based upon the physical viewpoint expressed in this paper led to the following equations for Momentum;

$$
p_{f}=\frac{m_{0} c_{0}}{\pi} U \int_{\beta=0}^{\beta=2 \pi} I 1
$$

(see equations (22) and (27)), and for Kinetic Energy;

$$
E_{K}=\frac{m_{0} c_{0}^{2}}{2 \pi} U \int_{\beta=0}^{\beta=2 \pi} I 2-m_{0} c_{0}^{2}
$$

(see equations (38), (41), and (42)). $U, I 1$, and $I 2$ are given by equations (25), (13), and (14) respectively, and the $I 1$ and $I 2$ integrals for specific values of $v_{f}$ are given in Table 1 and Table 3. Note that equation (51) predicts a rest energy which is equal to the relativistic rest energy.

These equations predict the Matter Wave Momentum and Kinetic Energy shown in Figures 3 and 4 respectively, as a function of speed. The agreement with experimental evidence is reasonable. As explained in section 5, the settings for both the momentum and kinetic energy proportionality constants required that the ratio of specific heats, used in the longitudinal sound wave model, be equal to 1 (ref, equation (43)). This indicates that the medium which bears longitudinal matter waves is not very compressible and behaves more like water than like a gas. Also, an unexpected result of the sound wave model used is that the matter wave amplitude squared over the cycle time increases with speed, as described by equations (20) and (25). This together with the possibility of a medium whose density may vary as little as that for longitudinal sound waves in water is worthy of further study.

The quantum particle model developed is consistent with experimental results for momentum and kinetic energy vs speed, without requiring an infinite stationary wavelength or a pulse rate that matches the electromagnetic clock retardation that occurs physically under the Lorentz/Poincare' interpretation of the special relativity equations.

Evidence for a physical interpretation of the waves related to quantum objects is given by Tony Hey and Patrick Walters (1989). They describe the well known total internal reflection that occurs when light passes from glass to air at an angle greater than or equal to the 'critical' angle and no light escapes into the air. They then say that although no light rays penetrate the air beyond the glass, "there is nonetheless some sort of wave disturbance in the air" and that when another parallel block of glass is brought close to the first one, the wave disturbance "begins to penetrate the second block and a transmitted ray of light appears! The closer the two blocks are brought together, the more light energy that reappears as a transmitted ray". It is not difficult to imagine that this wave disturbance extends into space and is physical, having little to do with probability.

As mentioned in section 6, an underlying assumption of the viewpoint taken in this paper is that because a quantum particle is considered to be nothing more than the sum of "in" and "out" matter waves focused through its center, whatever happens to these matter waves determines the future location of that center. It is hoped that other researchers will advance this assumption and develop physical explanations for gravity, interference, and the slowdown of light in transparent mediums. Gravity, for example, was briefly considered in section 6 to be caused by the local gradient in matter wave speed near a large body like earth. There we determined the wavelength difference, in the components of a harmonically oscillating pseudo standing wave, required for these components to pass through a particle center in the same cycle time.

\section{References}

De Broglie, L. (1925). On the Theory of Quanta. A translation of Recherches Sur La Theorie Des Quanta. Ann. De Phys., $10^{e}$ Serie t. III (Janvier-Fevrier 1925) by A.F. Kracklauer, Chapter 1. Retrieved from http://aflb.ensmp.fr/LDB-oeuvres/De_Broglie_Kracklauer.pdf

Galison, P. (2003). Einstein's Clocks, Poincare's Maps, p 210. New York, NY: W.W. Norton \& Company. 
Integral Calculator (2020). David Scherfgen - All rights reserved. Retrieved from https://www.integral-calculator.com

Bertozzi, W. (1964). Speed and Kinetic Energy of Relativistic Electrons. American Journal of Physics, 32(7), 551-555.

Hey, T., \& Walters, P. (1989). The Quantum Universe (pp. 55-57). New York, NY: Cambridge University Press.

Rogers, M., Mc Reynolds, A., \& Rogers, F, (1940, March 1). A determination of the Masses and Velocities of Three Radium B Beta-Particles: The Relativistic Mass of the Electron. The Physical Review, 57(5), 379-383.

Resnick \& Halliday (1962, April). Physics, Part I, pages 429-430. New York: John Wiley \& Sons, Inc.

Peskin, C. (2010, Aug 25). Wave Momentum. Courant Institute of Mathematical Sciences, New York University, 1-2. Retrieved from https://www.math.nyu.edu/faculty/peskin/papers/wave_momentum.pdf

Wagner, D. (2015, June). Fresnel vs. Einstein - a New Direct Test. Applied Physics Research, 7(3), 33-40. Retrieved from http://www.ccsenet.org/journal/index.php/apr/article/view/47570

\section{Copyrights}

Copyright for this article is retained by the author(s), with first publication rights granted to the journal.

This is an open-access article distributed under the terms and conditions of the Creative Commons Attribution license (http://creativecommons.org/licenses/by/4.0/). 